ERRATUM

\title{
Pediatric infratentorial gangliogliomas: a retrospective series
}

TO THE READERSHIP: An error appeared in the article by Baussart et al. (Baussart B, Di Rocca F, Garnett $\mathrm{MR}$, et al. Pediatric infratentorial gangliogliomas: a retrospective series. J Neurosurg. 2007;107[4 Suppl Pediatrics]: 286-291).

The first author's surname was incorrectly published as "Baussard." The correct spelling is "Baussart."

The article has been corrected online as of June 11, 2021.

Bertrand Baussart, MD, PhD

Hôpital Pitié-Salpétrière, AP-HP Sorbonne, Paris, France

INCLUDE WHEN CITING

Published online June 11, 2021; DOI: 10.3171/2021.5.PEDS2116748a.

CAANS 2021, except where prohibited by US copyright law 